\title{
DISEÑO DE UN DISPOSITIVO DE ILUMINACIÓN DE VÍAS PÚBLICAS CON POSICIONAMIENTO AUTOMÁTICO
}

\author{
DESIGN OF A SELF-POSITIONING STREET LIGHTING DEVICE \\ José Ángel Garrido ${ }^{a, b,{ }^{*}}$, Ángel Marqués ${ }^{b}$, Ángel Martín ${ }^{b}$, José Llorens ${ }^{a}$ \\ ${ }^{a}$ Departamento de I+D+i, TECHNOSUN S.L.U., C/ Villa de Madrid (P.I. Fuente del Jarro), 46988 Paterna, España. \\ angel.garrido@technosun.com; jolloma7@topo.upv.es
}

${ }^{\text {b }}$ Departamento de Ingeniería Cartográfica, Geodesia y Fotogrametría, Universitat Politècnica de València, Camino de Vera s/n, 46022 València, España. amarques@cgf.upv.es; aemartin@upvnet.upv.es

\begin{abstract}
:
Current trends in urban lighting design are focused on factors such as energy efficiency, environmental sustainability, and multi-service effectiveness. As in most urban facilities, geographic location is a key attribute in the management of urban lighting infrastructures, and although street lighting hardware based on loT devices allow users to connect multiple sensors, GNSS chips are rarely considered in hardware and software design. In this paper, we present the prototype of a lighting device intended for urban networks that allows positioning lampposts. The hardware equipment consists of four fundamental blocks: the BeagleBone Black (BBB) board, the energy manager board, the serial wired LED array, and the RS-485 communication bus. Blocks one and two form a subsystem that we name the energy management system (EMS), while block four is responsible for low level communication with the sensors, particularly the GNSS sensor. After its integration in the network, our device is considered as a node in a branched structure with three hierarchical levels: the operations command centre, the control cabinets, and the lampposts themselves; or in other words, the central, mid, and peripheral node groups respectively. The operation of the positioning procedure is mostly autonomous, starting with the GNSS data collection by the lamppost equipment. All those data are sent to the upper level, i.e. cabinets, for further processing in a different computing environment. This separation reduces the computational requirements of terminal nodes. Nodes in level 2 are also responsible for communicating geolocation updates to nodes in level 3 as well as to the command centre in level 1. Communication tasks rely on HTTP requests over lines that link the three levels. We used the open-source platform Fiware, a framework designed to support smart solutions, which in turn relies on other well-known components such as MongoDB, a NoSQL database system. As a result, we have a positioning procedure that can be embedded into a lighting network that guarantees efficiency and other requirements in modern city management.
\end{abstract}

Key words: renewable energy management, lighting networks, georeferencing, GNSS sensor, loT technology, IT

\section{Resumen:}

Las tendencias actuales en el diseño de iluminación urbana se centran en factores como la eficiencia energética, la sostenibilidad medioambiental y la eficacia de múltiples servicios. Como en la mayoría de las instalaciones urbanas, la ubicación geográfica es un atributo clave en la gestión de las infraestructuras de iluminación urbana, y aunque el hardware de alumbrado público basado en dispositivos loT permite a los usuarios conectar múltiples sensores, los chips GNSS rara vez se consideran en el diseño de hardware y software. En este trabajo presentamos el prototipo de un dispositivo de iluminación destinado a redes urbanas que permite geolocalizar farolas. El equipo de hardware consta de cuatro bloques fundamentales: la placa BeagleBone Black (BBB), la placa eletrónica de gestión de la energía, la matriz de LED cableados en serie y el bus de comunicación RS-485. Los bloques uno y dos forman un subsistema que denominamos sistema de gestión de energía (EMS), mientras que el bloque cuatro es el responsable de la comunicación de bajo nivel con los sensores, en particular el sensor GNSS. Tras su integración en la red, nuestro dispositivo es considerado como un nodo en una estructura ramificada con tres niveles jerárquicos: el centro de mando, los nodos de control y las propias farolas; o en otras palabras, los grupos de nodos central, medio y periférico, respectivamente. La operación del procedimiento de posicionamiento es fundamentalmente autónoma, comenzando con la recolección de datos GNSS por el equipo electrónico de la luminaria. Todos esos datos se envían al nivel superior para su posterior procesamiento en un entorno informático diferente. Esta separación reduce los requisitos computacionales de los nodos terminales. Los nodos en el nivel 2 también son responsables de comunicar las actualizaciones de geolocalización a los nodos en el nivel 3, así como al centro de mando en el nivel 1. Las tareas de comunicación se basan en solicitudes HTTP a través de líneas que vinculan los tres niveles. Utilizamos la plataforma de código abierto Fiware, un marco diseñado para admitir soluciones inteligentes, que a su vez se basa en otros componentes de código bien conocidos como MongoDB, un sistema de base de datos NoSQL. Como resultado, tenemos un procedimiento de posicionamiento que puede integrarse en una red de iluminación que garantiza la eficiencia y otros requisitos en la actual gestión de la ciudad.

Palabras clave: gestión de energías renovables, redes de iluminación, sensor GNSS, tecnología loT, TIC

*Corresponding Author: José Ángel Garrido, angel.garrido@technosun.com 


\section{Introducción}

Actualmente existe una tendencia ampliamente aceptada hacia el desarrollo de ciudades inteligentes basadas en los siguientes pilares (Albino et al. 2015):

- Uso de energías renovables y sistemas autosostenibles energéticamente.

- Gestión inteligente y eficiente de la energía necesaria para alimentar al sistema, tanto el alumbrado público como otros usos y servicios del espacio urbano.

- Georreferenciación de los elementos más importantes del alumbrado público, específicamente farolas y centros de mando.

- Asociación de sensores tanto para la monitorización de variables medioambientales, como de sensoresactuadores del propio sistema para el control, monitorización y fiabilidad del sistema.

- Conectividad mediante dispositivos Internet of Things (IOT) y tecnologías de la información y comunicación (TIC).

El uso combinado de estos cinco conceptos es la clave de la noción de Smart city del futuro.

Este artículo presenta los primeros resultados de una tesis doctoral desarrollada en modalidad de doctorado industrial en la empresa TECHNOSUN S.L.U. en el marco del programa de doctorado interuniversitario del departamento de Ingeniería Cartográfica, Geodesia y Fotogrametría de la Universitat Politècnica de València (UPV) y la Universidad Politécnica de Madrid (UPM). Esta tesis ha recibido fondos europeos a través de la Agència Valenciana de la Innovació AVI, cuyo objetivo es el fomento del $\mathrm{I}+\mathrm{D}+\mathrm{i}$ en el tejido industrial y empresarial Valenciano.

El objetivo de la tesis es contribuir a la gestión eficiente de energía en el contexto de la ciudad inteligente. La Figura 1 muestra un diagrama conceptual donde se representan todos los elementos que conforman el sistema en su totalidad.

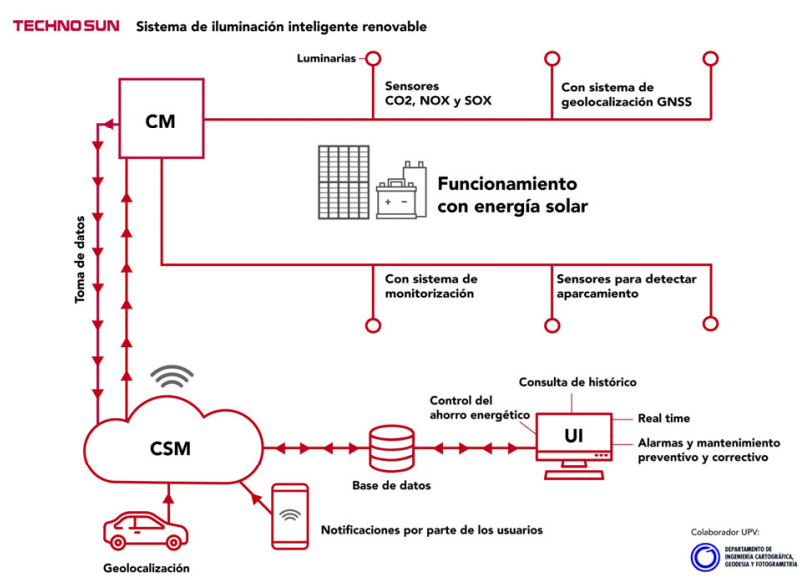

Figura 1: Bloques sistema completo.

La idea que se pretende transmitir en la Figura 1, es que el uso eficiente de una red de alumbrado público es un concepto básico para la ciudad inteligente. Esta red se puede dividir jerárquicamente en tres niveles: (1) sistema central de gestión (CMS), (2) centros o nodos de control
(CM) y (3) luminarias (nodos periféricos). Las luminarias en particular conforman un conjunto de puntos, repartidos por toda el área urbana, que potencialmente permiten recolectar, tanto datos de los sensores del sistema de iluminación, como datos relacionados con las variables medioambientales. Si dichas luminarias se equipan adecuadamente, permitirán además actuar sobre el comportamiento de las propias luminarias para gestionar la energía de manera eficiente, aparte de ayudar a monitorizar el entorno de manera autónoma.

Es importante que la componente tecnológica no nos haga perder de vista el propósito fundamental del concepto de ciudad inteligente, que es contribuir a la conservación del medioambiente e incrementar el bienestar y seguridad de todos los seres vivos, incluyendo los usuarios humanos, pero sin olvidar toda la diversidad tanto de animales como plantas que habitamos en nuestro planeta (Pérez 2019).

En este sentido cabe recordar que el planeta tierra es en última instancia un contenedor que posee unos recursos finitos: agua, energía y alimentos, animales, plantas y seres humanos. Sobre estos últimos recae la responsabilidad de preservar el entorno mediante la correcta gestión de los recursos y la cohabitación de forma respetable con toda la biodiversidad del planeta. Este enfoque vital es bien conocido en multitud de poblaciones indígenas y nuestro planeta recibe nombres propios como Pachamama en Sudamérica, Asase Yaa en algunas zonas de África o Akna en la cultura Inuit.

El proyecto de tesis doctoral que se está desarrollado presenta un grado de complejidad considerable como consecuencia del carácter multidisciplinar necesario para poder abordar, integrar y desarrollar todos los elementos que conforman el sistema.

En esta comunicación presentamos un primer prototipo funcional a pequeña escala en el que se considera un único nodo compuesto por:

- Luminaria, módulo solar, sistema de acumulación, sistema de control y monitorización con tres intensidades de regulación correspondientes a tres escalones de consumo en función de las necesidades.

- Caja de sensores. La caja asociada a este nodo contiene sensores GNSS para posicionamiento, además de sensores de temperatura y humedad. Asimismo, contiene el módulo solar, sistema de acumulación y sistema de control y monitorización al igual que en el caso anterior.

- $\quad$ Centro de mando. En nuestro prototipo, este bloque está compuesto por el dispositivo loT BeagleBone Black (nodo 2 intermedio) conectado a una red local, donde se encuentra integrado el equivalente del nodo de nivel 1 (sistema central), en una maquina con sistema operativo Linux, que en el prototipo definitivo se alojará en la nube haciendo uso del estándar Fiware que se explica posteriormente.

Este prototipo ya está funcionando y la interconexión entre luminarias-sistema fotovoltaico, caja de sensoressistema fotovoltaico integrado y centro de mando o $\mathrm{CM}$ se ha realizado mediante un bus de comunicaciones denominado Power over Bus RS-485 (POB-485). La Figura 2 muestra un diagrama de bloques del prototipo. 


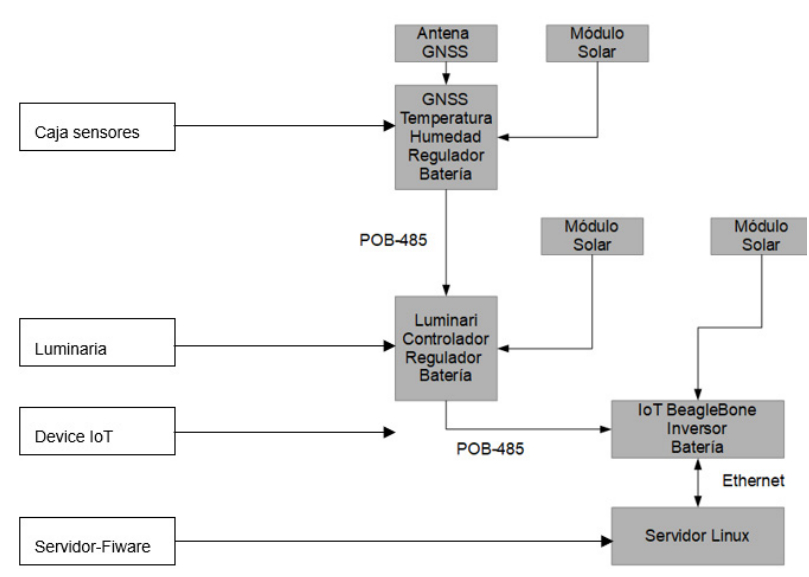

Figura 2: Bloques prototipo funcionando.

Es interesante destacar que tanto el dispositivo loT como el servidor Linux y la propia red local están alimentados por energía solar. Esto se implementa por medio de un inversor-sai que por defecto alimenta de forma ininterrumpida el sistema con energía fotovoltaica. En caso de fallo o agotamiento de las baterías debido a la ausencia de energía solar, el sistema toma la energía de la red eléctrica para mantener la continuidad del servicio. La autonomía nominal de este prototipo es de 10 días.

\section{Gestión energética}

Uno de los aspectos fundamentales de este trabajo es el enfoque en la gestión y uso de la energía eléctrica. El concepto de gestión energética tiene como punto de partida fundamental la orientación del diseño del sistema, tanto software como hardware, hacia los principios de uso eficiente, razonable y sostenible de la energía eléctrica. Esto se consigue a través de los siguientes elementos:

- Conversión en origen, en los centros de mando y control, de la corriente eléctrica alterna $(A C)$ trifásica en corriente continua (DC).

- Maximización de la producción de energía eléctrica procedente de fuentes renovables (tan cercana al consumo real de energía como sea posible).

- Optimización de las perdidas en las líneas de distribución del alumbrado.

- Optimización de la gestión y conversión de la energía punto a punto (luminaria hardware DC/DC BUCK).

Una vez alcanzados los puntos anteriores, se debe aplicar un control de la energía en función de las necesidades reales de cada una de las vías que alumbran las luminarias, adecuando la intensidad lumínica a dichos recursos necesarios y por tanto controlando el consumo energético.

En la situación operativa final, se trata de ejercer el control citado anteriormente en función de la ocupación o saturación de la vía y aplicando distintos tramos horarios en los que se regula la intensidad lumínica y por consiguiente el consumo.

\subsection{Conversión de $\mathrm{AC}$ a $\mathrm{DC}$ en los $\mathrm{CM}$}

El objetivo de esta transformación es elevar al máximo la tensión eléctrica y minimizar todo lo posible la corriente eléctrica que se transmite sobre las líneas. También es necesario eliminar la descompensación habitual de potencia entre las fases de la corriente alterna trifásica, evitando así la generación de perdidas en los centros de transformación por la descompensación de potencias entre fases donde se conectan los CM. La Figura 3 muestra el esquema eléctrico de la rectificación de $\mathrm{AC}$ a $\mathrm{DC}$ en el CM.

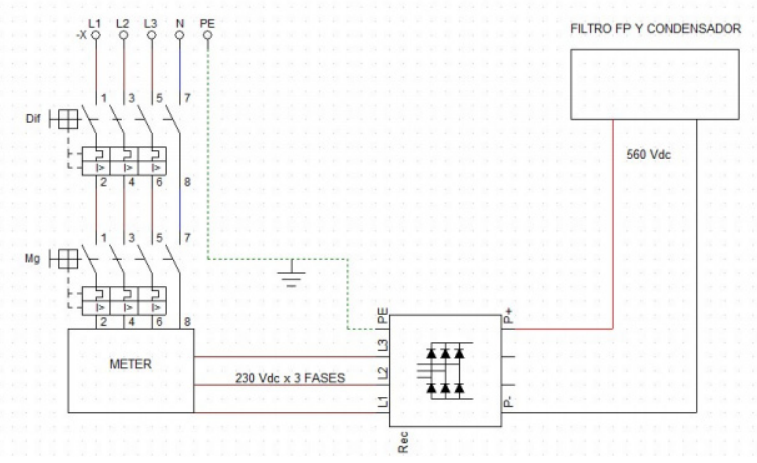

Figura 3: Esquema eléctrico rectificación y filtrado.

La Figura 4 muetra los componentes físicos que conforman la rectificación de $\mathrm{AC}$ a $\mathrm{DC}$ en el CM. La instalación está compuesta por diferentes bloques destinados a protección, monitorización de variables eléctricas, rectificador y filtro.

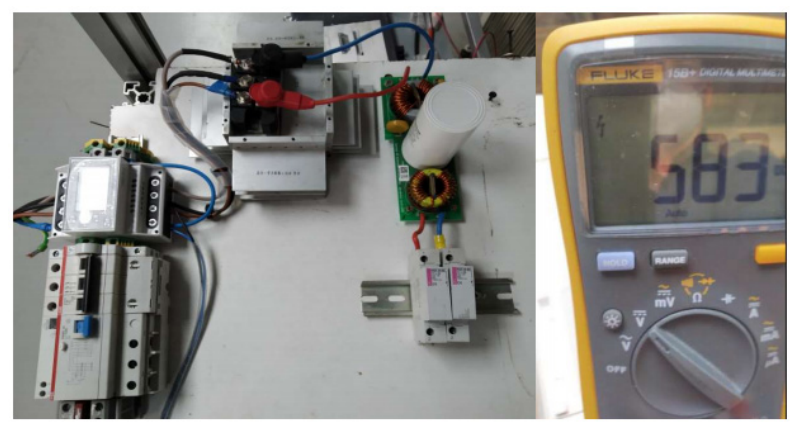

Figura 4: Componentes reales rectificador y filtro.

\subsection{Maximización en el punto de consumo del uso de energías renovables}

Para conseguir los puntos 2 y 3 citados anteriormente (maximización de la utilización de energía solar renovable y optimización de las perdidas en las líneas eléctricas), la premisa seguida durante el desarrollo e implementación del sistema es, generar energía fotovoltaica en los mismos nodos, sean periféricos o intermediarios. Como el consumo de dicha energía es nocturno, la producción energética no coincide con el consumo de energía siendo necesario la integración de los elementos: módulo solar, regulador de carga y batería o acumulador litio-ion.

De esta forma se consiguen dos propósitos importantes que conforman los pilares del sistema:

- Utilización de energía renovable.

- Disminución de las corrientes de línea minimizando pérdidas (una gran parte de la energía necesaria para los nodos, no tiene que ser transportada por las líneas ya se encuentra acumulada el las baterías de cada nodo). 
En la Figura 5, se muestran los elementos que conforman los nodos autónomos.

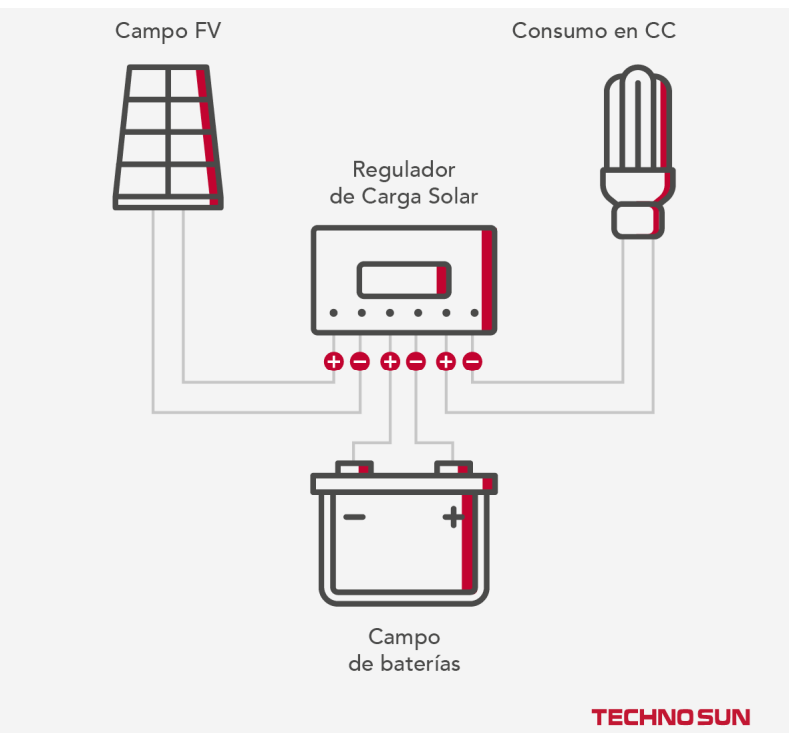

Figura 5: Elementos de los nodos autónomos.

\subsection{Minimización de las perdidas en las líneas de distribución del alumbrado}

Es necesario tener en cuenta que las pérdidas en las líneas se producen como consecuencia de la circulación de la corriente eléctrica, es decir, del total de la energía transportada hay un porcentaje de ésta que se pierde (o se malgasta) por efecto Joule en forma de calor (Joule 1884).

Parece bastante razonable que la magnitud sobre la que debe actuarse es la corriente eléctrica, con el objetivo de minimizarla. Este comportamiento es posible, por una parte, maximizando el trasnporte en forma de tensión (V) y no de corriente (I), y, por otra parte, consumiendo la energía en el mismo punto de generación, evitando así las perdidas como consecuencia de la resistencia del tendido eléctrico que aumenta con la longitud (L).

\subsection{Optimización de gestión y conversión de la energía punto a punto (luminaria hardware DC/DC BUCK)}

Se ha desarrollado una tarjeta electrónica que se encarga de la puesta en marcha, control de la potencia, y por lo tanto de la intensidad lumínica (gestión energética). La electrónica de la tarjeta consta de una fuente de alimentación conmutada Buck-Reductora de potencia, controlada analógicamente. La gestión se lleva acabo mediante la variación de los parámetros Ton y TofF (PWM $V_{R M S}$ en $D C$ ) de la tensión de alimentación del led en función de la tensión de entrada y las necesidades preestablecidas de consigna de tensión a corriente constante. Existe además un proceso de controlsupervisión digital que se ejerce a través de la BBB.

La potencia se ajusta en función de la variación de la tensión manteniendo la corriente constante. En su estado de operación, la tarjeta electrónica puede funcionar de forma indefinida mediante el control analógico sin necesidad de intervención del sistema digital. En caso de problemas del control de la intensidad lumínica o avería del controlador analógico, el sistema digital toma el control, quedando indicada la avería o anomalía sin interrupción del servicio.

En la Figura 6 se observa un diagrama de bloquesesquemático electrónico que muestra el conjunto de la tarjeta electrónica.

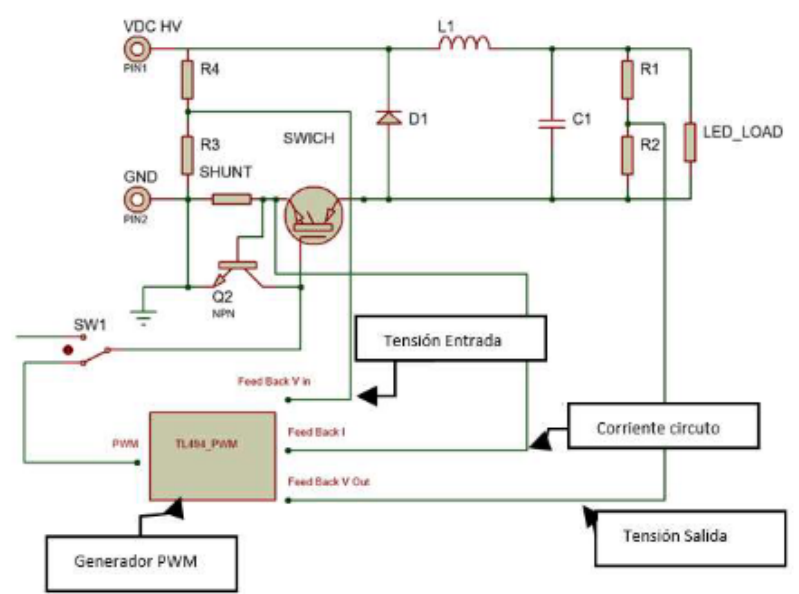

Figura 6: Esquema Fuente Buck-reductora.

La fuente descrita actúa sobre la luminaria (Fig. 7) aplicando alta tensión mediante la configuración de la trama LED gracias a la disposición en serie de 15 LEDs de $33 V_{D C}$, en total $495 V_{D C}$ y cuya corriente máxima es de $0.250 \mathrm{~A}$. La potencia total máxima es de $123 \mathrm{~W}$ en corriente contínua.

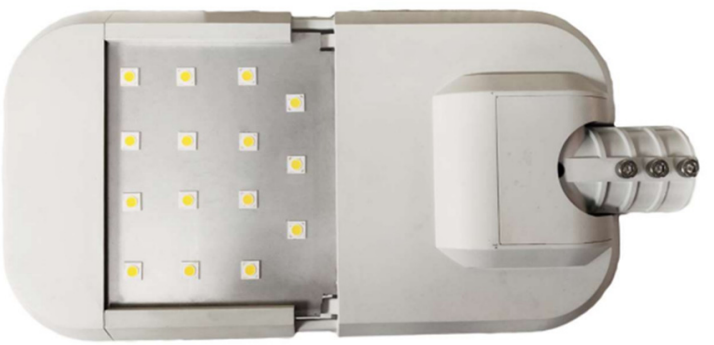

Figura 7: Luminaria de Alta Tensión.

Las ventajas de este sistema en términos de ahorro total son evidentes. Los elementos clave para la minimización de perdidas de energía eléctrica en el tendido eléctrico son la utilización de la tecnología led, la utilización de fuentes de alimentación conmutadas DC/DC Buck, generación y acumulación (Li-lon) de energía en el mismo punto de consumo, la fuente de generación energía solar fotovoltaica y finalmente la regulación y control de la intensidad lumínica y por lo tanto de la energía consumida.

Es interesante remarcar que en el diseño del prototipo final se entrega la potencia a la máxima tensión posible y a la menor corriente posible.

\section{Prototipo del sistema}

Los bloques que conforman el prototipo del artículo se muestran en la Figura 8 y se describen en esta Sección.

El prototipo diseñado consta de los siguientes subsistemas:

- Subconjunto caja de sensores. 
- Subconjunto luminaria.

- Dispositivo loT.

- Servidor Fiware sobre Linux Ubuntu.

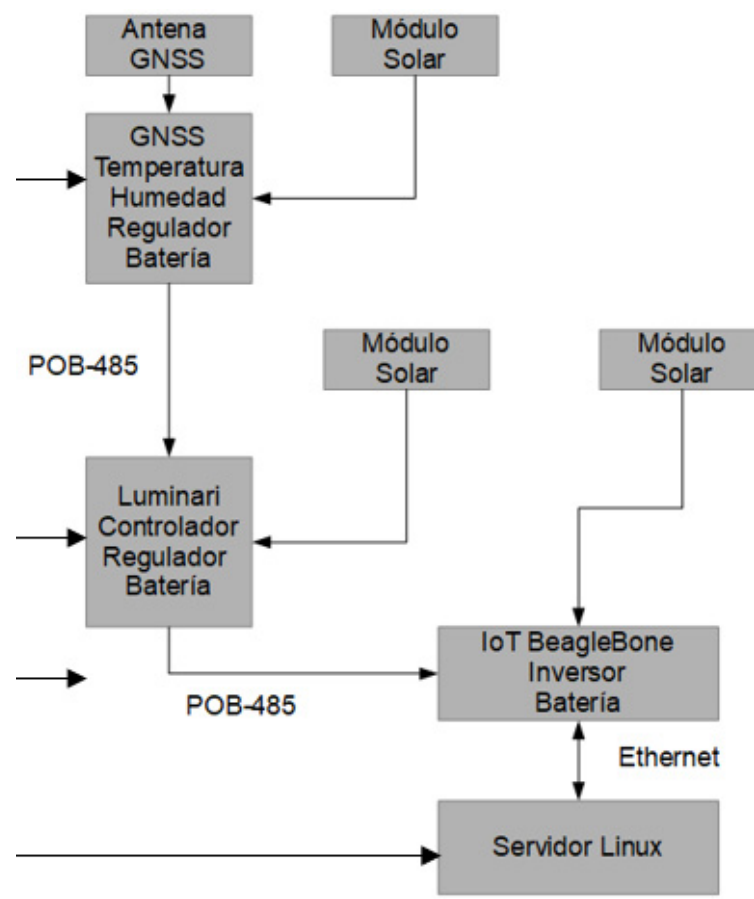

Figura 8: Bloques del prototipo.

\subsection{Subconjunto caja de sensores}

El sensor GNSS transmite los datos por el bus de comunicaciones serie RS-232 mediante el pin TX, que se conecta a su vez a un conversor serie RS-485. Este conversor permite dos acciones importantes, por una parte, prolongar la distancia de propagación de los datos hasta $1200 \mathrm{~m} \mathrm{y}$, por otra parte, poder conectar otros sensores o dispositivos al mismo bus. El bus es un recurso compartido multi-punto, cuyas salidas se llaman A+ y B-, a las cuales nos referiremos simplemente como bus compartido a partir de aquí.

El sensor de humedad y temperatura se conecta al dispositivo BBB a través del pin 2 dedicado a la entrada de interrupciones. La lectura de temperatura y humedad son gestionadas por una librería específica proporcionada por el fabricante mediante interrupciones en este pin. La placa BBB a su vez, se conecta a través del pin 1 TX RS-232 a otro conversor RS-232 a RS-485, conectando al bus compartido RS-485.

Se ha programado un software en lenguaje $C$, con un bucle de toma de datos bi-frecuencia. La operación normal consiste en recolectar datos GNSS y transmitirlos por el bus compartido. En periodos de 30 segundos el dispositivo BBB inhibe la línea del bus compartido RS485 del receptor GNSS y habilita el periférico RS-485 conectado a la propia BBB, emitiendo por el recurso compartido, la temperatura y la humedad, pasando a continuación a restaurar el envío de datos del sensor GNSS sin pérdida de datos. La habilitación-inhibición se consigue mediante dos salidas digitales.

El recurso compartido (formado por el POB-RS485 Power Over Bus RS-485) y el cable se prolongan hasta una distancia de $70 \mathrm{~m}$, donde está situada una segunda placa IoT BeagleBoard Black en el laboratorio de Technosun.

La alternancia de los dos intervalos de recolección de datos es temporizada y controlada con la habilitación e inhibición de los dos periféricos RS-485, mediante dos salidas digitales, a través del bus compartido.

Durante el ciclo de vida del programa, cuando se procesan los datos del intervalo 2 , además de enviar la información de temperatura y humedad se evalúa la puesta en marcha o la parada de un ventilador encargado de mantener la temperatura de la caja en valores razonables. En caso de detección de una temperatura en el interior de la caja mayor o igual a $35^{\circ} \mathrm{C}$, se activa el ventilador, el cual no se desactiva hasta que la temperatura descienda por debajo de los $30^{\circ} \mathrm{C}$ dejando un rango de histéresis de $5^{\circ} \mathrm{C}$, para evitar la puesta en marcha y parada contínua del ventilador y por tanto un consumo exagerado.

Los datos recolectados por el sistema, tanto los datos GNSS como los datos ambientales, se envían utilizando sentencias NMEA (ver Sección 4).

El contenido de la caja de sensores (Fig. 9) consta de los siguientes elementos:

- Módulo solar fotofoltaico $8 \mathrm{~W}$.

- Regulador PWM TECHNOSUN 14 A 12 Vdc.

- BeagleBoard.

- Batería de GEL AGM 12 Vdc 7Ah.

- Sensor de Humedad y temperatura.

Esta composición proporciona un sistema con funcionamiento autónomo y energéticamente autosuficiente.

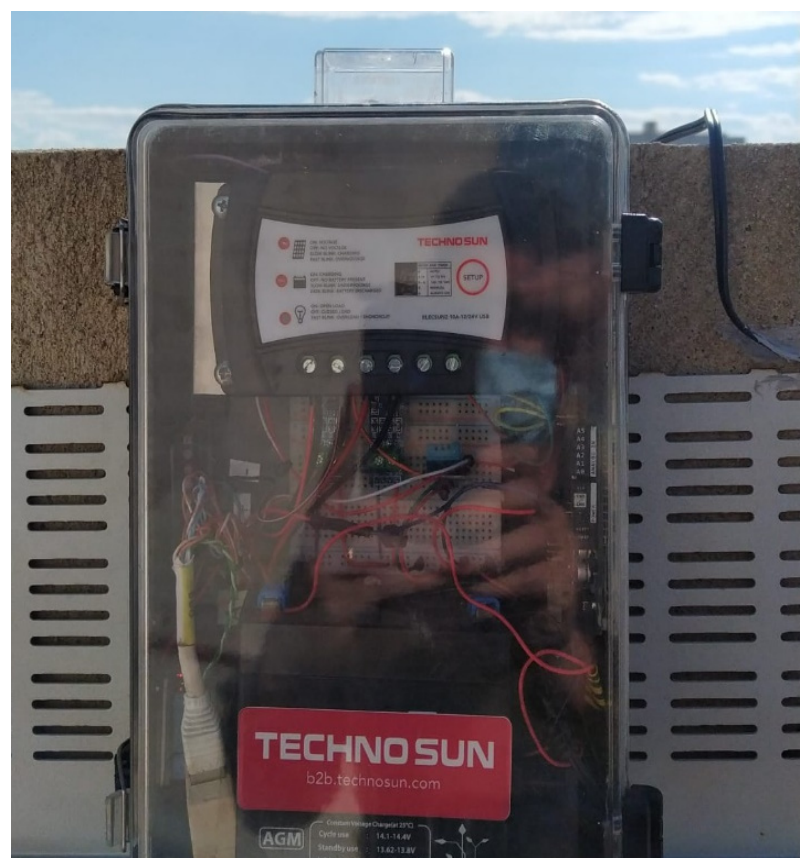

Figura 9: Caja de sensores instalada.

\subsection{Subconjunto luminaria}

La luminaria utilizada en el prototipo presentado en esta comunicación se observa en la Figura 10. La diferencia 
fundamental entre este prototipo y la luminaría con la que se está experimentando para la versión final de la tesis doctoral es que, a pesar de que las potencias son practicamente iguales, la forma en que se entrega dicha potencia es muy diferente. La Tabla 1 contiene una comparativa entre las características energéticas de las dos luminarias.

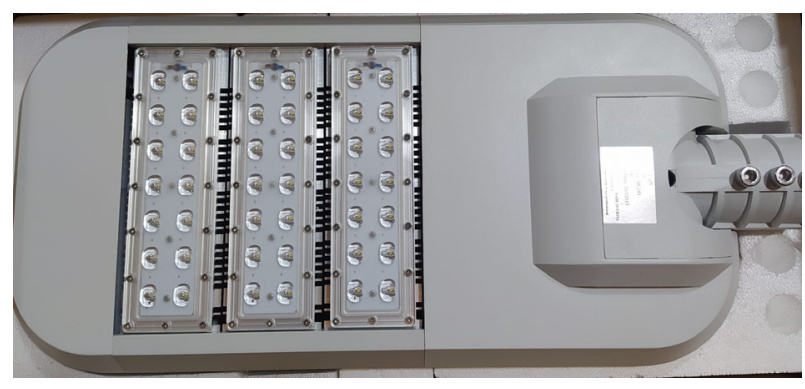

Figura 10: Luminaria de Baja tensión.

Tabla 1: Comparativa entre la implementación del prototipo presentado en $\mathrm{ClGeo} 2021$ y el prototipo final.

\begin{tabular}{c|c|c|c} 
Prototipo & Corriente $(A)$ & Voltaje $(V)$ & Potencia $(W)$ \\
\hline CIGeo & 10 & 12 & 120 \\
Final & 0.25 & 500 & 125
\end{tabular}

A pesar de tener potencias muy parecidas, en el caso del prototipo final, esta potencia es entregada aumentando la energía potencial $(V \times t)$ y disminuyento la energía cinética $(I \times t)$ al máximo. Este aspecto es de vital importancia, puesto que las perdidas de energía siempre se producen por el calor que se desprende como consecuencia del movimiento de los electrones. A mayor corriente mayor es la cantidad de electrones que circulan por el conductor y los propios LEDs, aumentando así la energía disipada en forma de calor y no de intensidad lumínica.

\subsection{Dispositivo loT}

El sistema empotrado BeagleBoard Black dispone de un microprocesador principal o host Sitara AM335x ARM A8 a $1 \mathrm{GHz}$ y dentro de este, dos unidades PRU (AM335x PRU-ICSS Reference Guide, Programmable Real-Time Unit), también denominadas DSP (Digital Signal Processor) que son las que se encargan de gestionar el procesado de señales digitales. Estas dos unidades pueden ser directamente programadas de forma independiente del host, o a través del sistema operativo Linux. El conjunto forma un Mini PC embebido con sistema operativo Debian Linux.

Se ha considerado como plataforma ideal para este proyecto porque combina en un mismo chip la posibilidad de programar a alto y bajo nivel. El usuario de lenguajes de alto nivel como C, Python, e incluso JavaScript mediante Node, pueden desarrollar código como en cualquier entorno Linux. Sin embargo, esta placa permite además la posibilidad de programar microcontroladores directamente, ya sea con librerías en lenguaje C especializadas o incluso en lenguaje ensamblador, siendo esta una visión más cercana al hardware a bajo nivel, muy útil para usuarios con perfil de ingeniero electrónico.
El diseño de la BBB permite entradas y salidas digitales, entradas de interrupción, entradas analógicas y salidas PWM, cubriendo así todas las necesidades desde el punto de vista de la electrónica.

Esta placa se podría definir como una hibridación entre un sistema basado en microprocesador con otro basado en microcontrolador, es decir, se puede considerar como un sistema microcontrolado con capacidades de trabajo en tiempo real.

\section{Recolección y transmisión de datos}

Las operaciones de recolección y transmisión de datos se han llevado a cabo mediante varias piezas de software que permiten el intercambio de información entre los distintos componentes físicos del sistema descrito en secciones anteriores.

El origen de los datos son los dos sensores integrados en el sistema, el sensor GNSS (NEO-6 de U-BLOX) y el sensor de variables ambientales (DHT 11 Humidity \& Temperature). Este sistema es asimilable a un nodo de nivel 3 (luminaria) en el esquema ramificado descrito anteriormente. Estos conjuntos de datos se transmiten a un nodo de nivel 2, equivalente a un gabinete de control en una red de iluminación real. En este trabajo, el nodo de nivel 2 consiste en un ordenador que actúa de intermediario con el espacio de almacenamiento final de los datos, o nodo de nivel 1. Este espacio de almacenamiento estaría en el dominio del centro de control en el diseño final y en el prototipo presentado aquí consiste en otro ordenador comunicado con el resto de nodos mediante una red Ethernet. En esta sección detallamos algunos aspectos del funcionamiento del conjunto.

El receptor GNSS es un modelo muy popular en muchas aplicaciones de electrónica amateur. Aunque se trata de un modelo de bajo coste, tiene la precisión suficiente para usarse en proyectos profesionales. Este receptor, como la mayoría de receptores de su categoría, proporciona una salida procesada estructurada en el protocolo de la National Marine Electronics Association (NMEA 1998). El protocolo NMEA define todos los aspectos técnicos de la transmisión de datos, desde la velocidad de transmisión recomendada, hasta el juego de caracteres utilizado o la terminología de uso para fabricantes. La información más relevante en este trabajo es la definición del formato de datos. En el formato NMEA, la información se transmite secuencialmente en fragmentos de texto denominados sentencias NMEA. Una sentencia está delimitada por un carácter inicial y dos caracteres finales. Entre estos delimitadores la información se estructura en campos separados por comas. Algunos campos están definidos con una longitud fija, aunque en general ésta puede ser variable. Es obligatorio que cada sentencia tenga un código de comprobación o ckecksum que es un campo especial situado al final de la sentencia y separado del resto de campos con un asterisco.

Un ejemplo de sentencia tomado de la publicación original (NMEA 1998) ayudará a entender el formato:

\$GPGLL, 5057.970 , N , $00146.110, \mathrm{E}, 142451, \mathrm{~A} * 27$ $<\mathrm{CR}><\mathrm{LF}>$

En este caso la etiqueta identificadora de la sentencia es GPGLL. Las sentencias transmitidas por receptores GPS/GNSS comienzan por las letras GP. En el caso 
particular de la sentencia GPGLL, la información transmitida consta de las coordenadas geográficas latitud y longitud, la hora del cálculo de la posición y el estado del sistema. En este caso el código de verificación es 27 y está separado del resto de información con un asterisco.

Los delimitadores de la sentencia son el símbolo $\$$ al comienzo y los caracteres CR (retorno de carro) y LF (nueva línea) al final. Estos dos últimos caracteres no son imprimibles y por tanto son invisibles en un listado NMEA.

La comunicación entre el receptor GNSS integrado en la caja de sensores, y el ordenador que simula el nodo de nivel 2 es inmediata y se realiza a través del bus RS-485 compartido. En el nodo de nivel 2 existe un programa que recibe los datos transmitidos por el puerto serie y realiza una serie de transformaciones de formato con el objetivo de agrupar la información de la manera más adecuada para las posteriores etapas del procesamiento. Estos dos elementos fundamentales de la comunicación, el receptor GNSS y el nodo superior, se denominan talker y listener en terminología NMEA.

El software en ejecución en el nodo de nivel 2 consiste en un script Python que hace uso de un módulo llamado nmea, desarrollado específicamente para esta tesis doctoral. El módulo contiene funciones específicas para la decodificación de las sentencias NMEA originales y su transformación a otra estructura de datos basadas en diccionarios. En la versión actual únicamente es posible decodificar las sentencias GPGGA y GPGSV, que en realidad cubren las necesidades de posicionamiento requeridas. La sentencia GPGGA es similar a la GPGLL, pero contiene algunos parámetros interesantes como el HDOP y un indicador de calidad no presentes en esta última. Por su parte, la sentencia GPGSV contiene información sobre los satélites usados en el cálculo de las coordenadas que podría ser usada en etapas de postproceso.

El módulo nmea fusiona la información de las sentencias GPGGA y GPGSV y crea un diccionario intermedio con la información relevante. Este diccionario se procesa posteriormente para su almacenamiento en disco.

El sensor de temperatura y humedad también envía datos desde la caja de sensores hasta el nodo de nivel 2 . En la Sección 3 se describió el mecanismo para compartir el mismo bus de datos entre los dos sensores. Sin embargo, este sensor no proporciona salida en formato NMEA, lo cual es un inconveniente a la hora de integrar la información de ambos sensores.

La solución que adoptamos fue crear una sentencia específica para transmitir datos ambientales. El propio estándar NMEA proporciona un esquema para crear este tipo de sentencias, llamadas sentencias propietarias, de modo que un fabricante pueda poner a disposición del usuario final toda la información relevante de su dispositivo (NMEA 1998).

Las etiquetas de las sentencias propietarias comienzan por la letra $P$ y van seguidas de tres caracteres que identifican el fabricante del equipo y uno o varios caracteres finales que identifican el tipo de información contenida en la sentencia. En el equipo descrito en esta comunicación creamos una sentencia para la transmisión de los valores de temperatura y humedad del interior de la caja de sensores, junto con el estado del ventilador. Un ejemplo de la sentencia propietaria es el siguiente:

\$PTSNE, $\left.32.2,45, \mathrm{OFF}^{*} 23<\mathrm{CR}\right\rangle\langle\mathrm{LF}\rangle$

Donde, siguiendo la recomendación del estándar, $\mathrm{P}$ indica sentencia propietaria, TSN es la abreviatura del nombre Technosun y $\mathrm{E}$ indica valores de parámetros ambientales. El significado de los campos es trivial, conteniendo valores de temperatura y humedad relativa de $32.2^{\circ} \mathrm{C}$ y $45 \%$ respectivamente, junto con el estado del ventilador que en este caso está desactivado. La sentencia se completa con el código de control o checksum y los terminadores de registro estándar.

La creación de la sentencia propietaria se lleva a cabo en tres fases. En primer lugar, se obtienen los valores numéricos en crudo y se convierten a variables de algún tipo válido del lenguaje $C$, lo que permite generar la sección de sentencia situada a la izquierda del asterisco. A continuación, se calcula el código de verificación con una función específica para tal fin. Finalmente, se añade el código calculado junto con los terminadores y la sentencia se envía por el bus compartido.

Las sentencias propietarias se intercalan entre las sentencias propias del receptor GNSS. Para ello, el microcontrolador del nodo final suspende temporalmente la salida procedente del receptor y envía la sentencia propietaria descrita anteriormente (ver Sección 3.1). En las pruebas que hemos realizado no se produce ninguna pérdida de datos importante, aunque a veces puede llegar alguna sentencia del receptor GNSS incompleta o con caracteres inservibles. En ese caso, el módulo de procesamiento NMEA ignora las sentencias corruptas.

La última etapa en el procesamiento de los datos procedentes de los sensores es el almacenamiento físico. Esta tesis se desarrolla en el contexto de las llamadas ciudades inteligentes y hemos optado por utilizar una plataforma especialmente creada para tales entornos llamada FIWARE (EC 2017; García 2015). Se trata de una plataforma respaldada por la Unión Europea que nació a partir de una iniciativa publico-privada para definir nuevas estrategias de emprendimiento alrededor de conceptos como los datos abiertos, las ciudades inteligentes o la Internet del futuro.

FIWARE se distribuye bajo el esquema de código abierto y consta de varios componentes que, a su vez, son ejemplos conocidos de la filosofía del código abierto. Un punto fundamental del sistema es su facilidad de uso, basada en un estándar universalmente aceptado hoy día como el protocolo HTTP (Fielding et al. 1999).

FIWARE tiene dos componentes principales. El primer componente se denomina Orion y es un intermediario que se encarga de comunicar los usuarios con el espacio de almacenamiento. El segundo componente es MongoDB (Hows et al. 2014), una base de datos NoSQL que se adapta perfectamente a las necesidades de escalabilidad y flexibilidad de una ciudad inteligente.

La instalación típica se basa en el uso de contenedores mediante Docker (Spencer 2016), aunque no es necesario usar este entorno para desplegar FIWARE. La instalación con Docker permite una gestión ágil de la plataforma y una resolución de problemas adecuada.

El almacenamiento en FIWARE se realiza desde el módulo nmea reseñado en esta misma sección. El 
mecanismo consiste en la ejecución de una función que toma como entrada los diccionarios intermedios creados a partir de la combinación de las sentencias NMEA procedentes del receptor GNSS y devuelve la información en el formato NGSI-LD (Privat 2021) utilizado por FIWARE.

Este formato se basa en un esquema JavaScript Object Notation (JSON) con una serie de requerimientos en cuanto al nombre y estructura de los campos. La transformación consiste básicamente en reformatear los diccionarios intermedios a cadenas JSON y enviarlos a Orion mediante una petición POST del protocolo HTTP.

Existen dos campos obligatorios en FIWARE llamados "id" y "type". El campo id debe ser único y tiene estructura URN (uniform resource name), con campos separados por el símbolo dos puntos (“:”). Un ejemplo de las pruebas realizadas es el siguiente:

"urn:ngsi-

ld: Technosun:001: GeolocationNMEAObserved: 2021-05-10T13:58:52.00Z"

Este identificador se representa en realidad en una sola línea; en este caso se divide en dos líneas debido a limitaciones de formato de texto.

Los dos primeros campos del identificador, urn y ngsi-ld son estándar, mientras que el resto los define el usuario para garantizar un identificador único. El tercer campo representa la empresa donde se desarrolla la tesis doctoral. El cuarto campo es el identificador de la luminaria. El quinto es el tipo de la entidad FIWARE y por último se añade la fecha y hora de cálculo de las coordenadas. Este campo prodece de las sentencias NMEA procesadas y está expresado en tiempo UTC.

El otro campo obligatorio en cualquier entidad FIWARE es type. Este campo se define por el usuario y debe ser único en el dominio de la aplicación. En este trabajo hemos elegido el tipo "GeolocationNMEAObserved", que como hemos visto también se incluye por convenio en el identificador de las entidades.

El resto de campos que componen la entidad FIWARE son definidos por el usuario, mediante un nombre, un tipo y un valor encapsulados en una subcadena JSON. También es posible añadir metadatos a cada campo de la entidad. Un ejemplo de subcadena JSON para almacenar la latitud es el siguiente (la cadena se ha tabulado y separado en líneas para su mejor representación):

$$
\begin{aligned}
& \text { "lat": \{ } \\
& \text { "type": "Float", } \\
& \text { "value": } 39.517173833, \\
& \text { "metadata": \{\}} \\
& \text { \} }
\end{aligned}
$$

La concatenación de los campos "id", "type", "lat" y el resto de información forman una entidad FIWARE típica en formato JSON.

El resultado inmediato de las pruebas realizadas hasta ahora es el posicionamiento de la caja de sensores. La Figura 11 muestra la posición del dispositivo obtenida en las primeras pruebas del sistema sobre una imagen aérea.

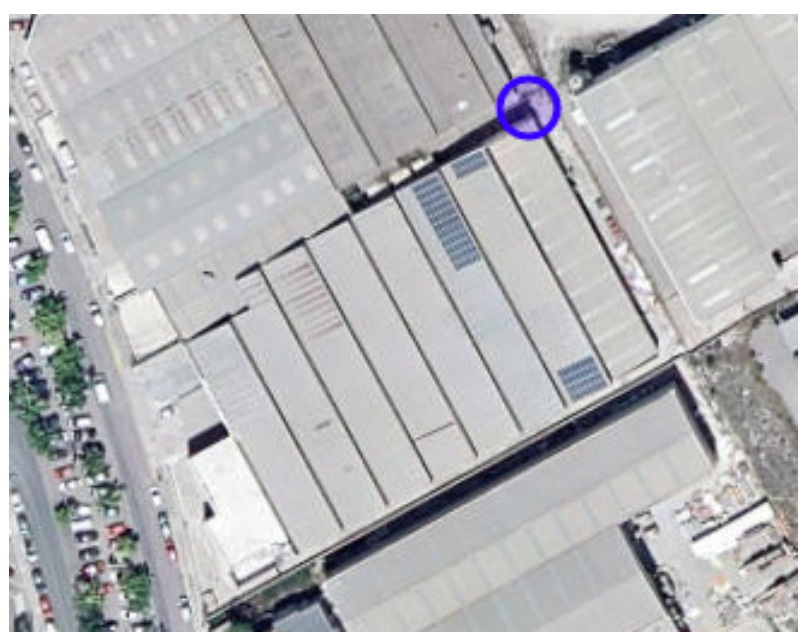

Figura 11: Posicionamiento de la caja de sensores.

\section{Conclusiones}

El trabajo presentado en esta comunicación ha permitido crear una aproximación cercana al prototipo final, así como la verificación de la solidez de los principios sobre los que se implementará el sistema completo.

Las pruebas realizadas hasta ahora han sido muy satisfactorias demostrando que el sistema es estable, tanto desde el punto de vista hardware como software.

La incorporación a cada nodo de un sistema de geolocalización basado en receptores GNSS, abre un nuevo camino en la georreferenciación de los componentes de la ciudad inteligente. Asimismo, el sistema de comunicación utilizado para la transmisión de datos constituye una infraestructura consistente que añade nuevas posibilidades, no solo para funciones de georreferenciación de los elementos que conforman el sistema, sino también para poder suministrar servicios geolocalizados a terceros en un futuro.

Por otra parte, la utilización de las energías renovables nodo a nodo presenta un ahorro importante del uso de energía eléctrica proveniente de fuentes convencionales no renovables.

Por último, otro aspecto de diseño importante es el uso de corriente continua, en contraposición al uso habitual de corriente alterna, y particularmente la forma en que se transporta ésta a alta tensión, consiguiendo disminuir la corriente que fluye por las líneas y por tanto minimizando las pérdidas en el transporte.

\section{Agradecimientos}

El trabajo presentado en esta comunicación ha sido parcialmente financiado por la Agència Valenciana de la Investigació (AVI) mediante la concesión de la ayuda INNTA3/2020/26 del Programa Promoción del talento L3, doctorandos empresariales (Innodocto).

También quiero agradecer a TECHNOSUN S.L.U., y en especial a los CEOS Antonio Ramos Beneyto y Toni Ramos Sánchez por la confianza depositada tanto en esta tesis doctoral, como en mí mismo. Asimismo, quiero agradecer la ayuda e ideas aportadas por el Ingeniero Eléctrico Jesús Saiz Crespo, compañero de trabajo en TECHNOSUN S.L.U 


\section{References}

ALBINO, V., BERARDI, U., and DANGELICO, R.M., 2015. Smart Cities: Definitions, Dimensions, Performance, and Initiatives. Journal of Urban Technology, 22, pp. 1-19.

JOULE, J.P., 1884. The scientific papers of John Prescott Joule. London: The Physical Society of London, 657 pages.

EC., 2017. Breaking the mould: A new model for EU innovation programmes. Brussels: European Comission, 72 pages.

FIELDING, R., GETTYS, J., MOGUL, J.C., FRYSTYK, H., MASINTER, L., LEACH, P. and BERNERS-LEE, T., 1999. Hypertext Transfer Protocol - HTTP/1.1. Washington DC: The Internet Society. 114 pages.

HOWS, D., MEMBREY, P., and PLUGGE, E., 2014. MongoDB basics. New York: APRESS, 120 pages.

GARCÍA, M., 2015. New businesses around open data, smart cities and FIWARE. Brussels: European Public Sector Information (EPSI) Platform. 30 pages.

NMEA., 1998. NMEA 0183 Standard for interfacing marine electronic devices. Version 2.30. New Bern: National Marine Electronics Association. 81 pages.

PRIVAT, G., 2021. Guidelines for modelling with NGSI-LD. ETSI White Paper No. 42. Nice: European Telecommunications Standards Institute. 54 pages.

PÉREZ, J.L., 2019. El poshumanismo. Los derechos de los seres vivos. La naturaleza y la humanidad en el Horizonte 2050. Madrid: Instituto Español de Estudios Estratégicos. 46 páginas.

SPENCER, A., 2016. Docker: The complete beginners guide to start with Docker. Scotts Valley: CreateSpace Independent Publishing Platform. 44 pages. 